\title{
SNHG8 is identified as a key regulator of epstein-barr virus(EBV)-associated gastric cancer by an integrative analysis of IncRNA and mRNA expression
}

\author{
Tao Huang ${ }^{1,2, *}$, Yan $\mathrm{Ji}^{2, *}$, Dan Hu${ }^{1, *}$, Baozheng Chen ${ }^{1}$, Hejun Zhang ${ }^{1}$, Chao Li ${ }^{1}$, Gang \\ Chen ${ }^{1}$, Xingguang Luo ${ }^{3}$, Xiong-wei Zheng ${ }^{1,4}$, Xiandong Lin ${ }^{1,4}$ \\ ${ }^{1}$ Department of Pathology, Fujian Provincial Cancer Hospital and Fujian Medical University Cancer Hospital, Fuzhou, Fujian, China \\ ${ }^{2}$ Institute of Health Sciences, Shanghai Institutes for Biological Sciences, Chinese Academy of Sciences and Shanghai Jiao \\ Tong University School of Medicine, Shanghai, China \\ ${ }^{3}$ Department of Psychiatry, Yale University School of Medicine, New Haven, CT, USA \\ ${ }^{4}$ Fujian Provincial Key Laboratory of Translational Cancer Medicine, Fuzhou, Fujian, China \\ ${ }^{*}$ Co-first authors
}

Correspondence to: Xiong-wei Zheng, email: agu1960@126.com Xiandong Lin, email: linxdong1970@yeah.net

Keywords: gastric cancer, epstein-barr virus, long non-coding RNA, biomarker, SNHG8

Received: July 10, 2016

Accepted: October 31, 2016

Published: November 07, 2016

\section{ABSTRACT}

The Epstein-Barr virus (EBV) is associated with a variety of cancers, including gastric cancer, which has one of the highest mortality rates of all human cancers. Long non-coding RNAs (IncRNAs) have been suggested to have important causal roles in gastric cancer. However, the interaction between IncRNAs and EBV has not yet been studied. To this end, we sequenced 11,311 IncRNAs and 144,826 protein-coding transcripts from four types of tissue: one non-EBV-infected gastric carcinoma (EBVnGC) and its adjacent normal tissue, and one EBV-associated gastric carcinoma (EBVaGC) and its adjacent normal tissue. Five IncRNAs showed EBVaGCspecific expression; of those, one (SNHG8) was validated using real-time PCR in an independent cohort with 88 paired gastric cancer and adjacent tissue samples. To explore the functions of SNHG8, we identified its mRNA targets on the IncRNA-mRNA co-expression network of the Illumina Body Map, which contains the RNA sequencing data of mRNAs and IncRNAs from 16 normal human tissues. SNHG8 IncRNA was found to affect several gastric cancer-specific pathways and target genes of EBV. Our results reveal the intertwined tumorigenesis mechanisms of IncRNA and EBV and identify SNHG8 as a highly possible candidate biomarker and drug target of gastric cancer.

\section{INTRODUCTION}

Gastric cancer is the fourth most common cancer worldwide and ranks second on the cause list of cancer death. [1]. It is a complex and highly heterogeneous disease. One type of gastric cancer is Epstein-Barr virus (EBV)-associated gastric carcinoma (EBVaGC), which constitutes almost a tenth of all gastric carcinomas [2]. EBV is absent in noncancerous mucosa but present in all cancer cells, and has a clonal nature in neoplastic cells; therefore, it is considered to have a causal role in gastric carcinoma [2, 3]. EBVaGC has been well-characterized molecularly and genomically [4]. However, the pathogenic mechanism of EBVaGC remains poorly understood.
Long non-coding RNAs (lncRNAs) are with $\geq 200$ nt but without open reading frames (ORFs). Many studies have demonstrated that lncRNAs have diverse biological functions, such as regulating epigenetic modulation, transcription, and translation [5, 6], and that they are dysregulated in various cancers [7-10]. Furthermore, lncRNAs are being increasingly recognized as master regulators of cancer $[6,8,11]$.

In gastric cancer, IncRNA dysregulation is associated with larger tumors, greater tumor invasion, more widespread metastasis, and lower survival rates $[12,13]$. For example, expression of the lncRNA PANDAR (promoter of CDKN1A antisense DNA damage activated RNA) is greater in cancerous tissue than in adjacent 
healthy tissue, and ectopic expression of this promoter is associated with various measures of cancer severity [14]. Another study showed that overexpression of the lncRNA H19 promoted proliferation, migration, invasion, and metastasis of gastric cancer [15]. However, there have been no reports investigating lncRNAs in EBVaGC.

Deep sequencing is a high-throughput technique that enables rapid and comprehensive exploration of a large number of lncRNAs and can be used to identify sequence variations and discover novel lncRNAs [16, 17]. Here, we used deep sequencing to examine the lncRNAs and protein-coding transcripts in four samples: EBVaGC and its adjacent normal tissue, and non-EBV-infected gastric cancer tissue (EBVnGC) and its adjacent normal tissue. Five lncRNAs were specifically expressed in EBVaGC. Analysis of lncRNA and mRNA co-expression and virus-host interactions revealed that the lncRNA SNHG8 interacts with EBV proteins and regulates several important target genes that affect downstream cancer pathways.

\section{RESULTS}

\section{EBVaGC definition}

EBVaGC is a lymphoepithelioma-like, diffusetype carcinoma with dense lymphocytic infiltration. It is identified by the expression of EBV-encoded small ribonucleic acid 1 (EBER1) in cancer cell nuclei, using in situ hybridization. Lymphoid stroma surrounds the EBER1-positive nuclei (Figure 1).

\section{Whole genome IncRNA and mRNA expression profiles}

Ultra-high-depth RNA sequencing data sets were generated from two tumor samples (EBVaGC sample with 70.6 million pair-end reads; EBVnGC sample with 65.2 million pair-end reads) and two matched normal samples (EBVaGC adjacent sample with75.5 million pair-end reads; EBVnGC adjacent sample with 53.5 million pair-end reads).

A Trim Galore! Cutadapt wrapper (v1.9.dev6) was used to trim raw sequencing reads, and low quality bases ( $<$ Q20) were removed using Trimmomatic v0.32 [18]. FastQC v0.11.3 was used to evaluate the qualities of the raw sequencing data and the trimmed data by analyzing base quality, GC content and sequence length distribution. TopHat2 v2.1.1 [19] was used to align the trimmed reads to the human genome (GRCh37) with reference annotation from Gencode v19. More than $90 \%$ of reads were mapped and over $85 \%$ of reads were uniquely mapped. rRNA genes were masked, and Cufflinks v.2.2.1 [20] was used to generate transcriptome assemblies. Transcriptlevel expression was measured as FPKM (fragments per kilobase of exon per million fragments mapped). The average numbers of expressed genes and transcripts $($ FPKM $>1)$ were 14,360 and 24,505, respectively.

\section{EBV-specific IncRNAs}

The lncRNAs specifically expressed in EBVaGC tissue were identified using criteria of $\geq 5$-fold change between FPKM values of the EBVaGC sample and the other three tissues (EBVnGC sample, EBVaGC adjacent sample, EBVnGC adjacent sample). Five EBVaGCspecific lncRNAs were identified: RNU12, H19, SNHG8, RP11-359D14.3, and MIR143HG (Table 1).

\section{Polymerase chain reaction validation of the EBV-specific IncRNA SNHG8}

Real-time reverse transcription polymerase chain reaction (RT-PCR) analysis was used to further validate the expression levels of the five EBV-specific lncRNAs in a cohort of 88 patients with gastric cancer, with primers designed in Primer Premier 5.0 software (Supplementary Table S1). The RP11-359D14.3 primer was difficult to design and was removed after unsatisfactory quality control results. There were no pathological differences between the gastric carcinoma samples used for RNA sequencing and those used for RT-PCR validation (Supplementary Table S2). SNHG8 expression was concordant with the lncRNA sequencing assay. The RT-PCR results of non-significant lncRNAs, RNU12, H19 and MIR143HG are shown in Supplementary Figures S1-S3. Notably, SNHG8 expression in EBVaGC was significantly higher than in EBVnGC (Figure 2) and in EBVaGC adjacent tissue (Figure 3).

\section{Biological functions of SNHG8 target genes}

Next, we identified the target mRNAs of SNHG8 by analyzing its coexpression with mRNAs according to the Illumina Body Map dataset. Using the online gene function annotation tool DAVID [21], which includes numerous annotation categories such as Gene Ontology (GO) and KEGG Pathways, we explored the functions of the target genes of SNHG8 (Table 2). These results indicated that SNHG8 targets pathways such as hsa03010 (ribosome), GO:0006412 (translation), GO:0045449 (regulation of transcription), GO:0006350 (transcription), GO:0008380 (RNA splicing), GO:0016071 (mRNA metabolic process), GO:0008134 (transcription factor binding), GO:0003677 (DNA binding), and GO:0030528 (transcription regulator activity). Notably, many of these functions, such as "transcription" and "mRNA metabolic process", were wellknown pathways or processes targeted by EBV [22-24].

\section{Relationship between SNHG8 and EBV in EBVaGC}

We further investigated the relationship between SNHG8 and EBV by analyzing the co-expression between host human mRNAs and EBV mRNAs. We used an EBV genomics dataset to identify target mRNAs of EBV genes. 
Table 1: The FPKM expression levels of EBV-specific IncRNAs

\begin{tabular}{|c|c|c|c|c|c|}
\hline Transcript ID & Transcript Name & $\begin{array}{c}\text { EBV-negative } \\
\text { tumor sample } \\
\text { (EBVnGC) }\end{array}$ & $\begin{array}{c}\text { EBV-negative } \\
\text { adjacent sample }\end{array}$ & $\begin{array}{c}\text { EBV-positive } \\
\text { tumor sample } \\
\text { (EBVaGC) }\end{array}$ & $\begin{array}{l}\text { EBV-positive } \\
\text { adjacent sample }\end{array}$ \\
\hline ENST00000362512 & RNU12 & 321.19 & 138.32 & 1648.37 & 264.21 \\
\hline ENST00000414790 & H19 & 0.49 & 1.99 & 30.63 & 0.98 \\
\hline ENST00000412788 & H19 & 0.00 & 0.00 & 6.73 & 0.52 \\
\hline ENST00000449007 & RP11-359D14.3 & 0.00 & 0.00 & 5.19 & 0.00 \\
\hline ENST00000384096 & SNHG8 & 0.00 & 0.00 & 4.21 & 0.01 \\
\hline ENST00000522358 & MIR143HG & 0.24 & 0.37 & 3.03 & 0.00 \\
\hline
\end{tabular}

Using the hypergeometric statistical test, we evaluated the overlap between EBV target genes and SNHG8 target genes. The enrichment results (Table 3) show that SNHG8 interacts significantly with EBV genes such as $B H L F 1, L F 3$, $B H R F 1$, and BNLF2a.

The EBV genes $B H L F 1$ (BamHI H leftward reading frame 1) and $L F 3$ (leftward reading frame 3) consist of repetitive sequences of 125 and $102 \mathrm{bp}$, respectively. They are both found in the polyribosomal fraction of cells infected with EBV and expressed transcriptionally in virusassociated tumors [25]. There is evidence that $B H L F 1$ and $L F 3$ are associated with the lytic replication cycle, which seems to take place mainly in epithelial cells. This type of replication is essential for the spread of the virus, and its suppression maintains the tumor phenotype [26].

BHRF1 has $38 \%$ primary sequence homology with the antiapoptotic protein $\mathrm{Bcl}-2$, and shares three of its four conserved regions (Bcl-2 homology $(\mathrm{BH})$ domains, BH1-BH3) [27]. The functions of BHRF1 are also similar to those of Bcl-2, and it imparts anti-apoptotic protection to EBV-infected cells [28], allowing the development of virus persistence and facilitating oncogenesis.

$B N L F 2 a$ is another early lytic gene, and encodes a tail-anchored protein situated in the membrane of the endoplasmic reticulum [29]. The BNLF2a protein has two domains: a hydrophilic, cytosolic $\mathrm{N}$-terminal domain and a hydrophobic, membrane-spanning C-terminal domain [30]. Both domains are required for immune escape, which involves the disruption of viral peptide transport into the endoplasmic reticulum and of peptide loading onto human leukocyte antigen class I molecules; this disruption leads to lower levels of endogenous antigen presentation, thus preventing recognition by CD $8+\mathrm{T}$-cells $[30,31]$.

\section{EBV target genes with the same expression pattern as SNHG8}

The target genes of EBV that are also targeted by SNHG8 are listed in Table 3. As described above, SNHG8 expression in EBVaGC was significantly higher than in
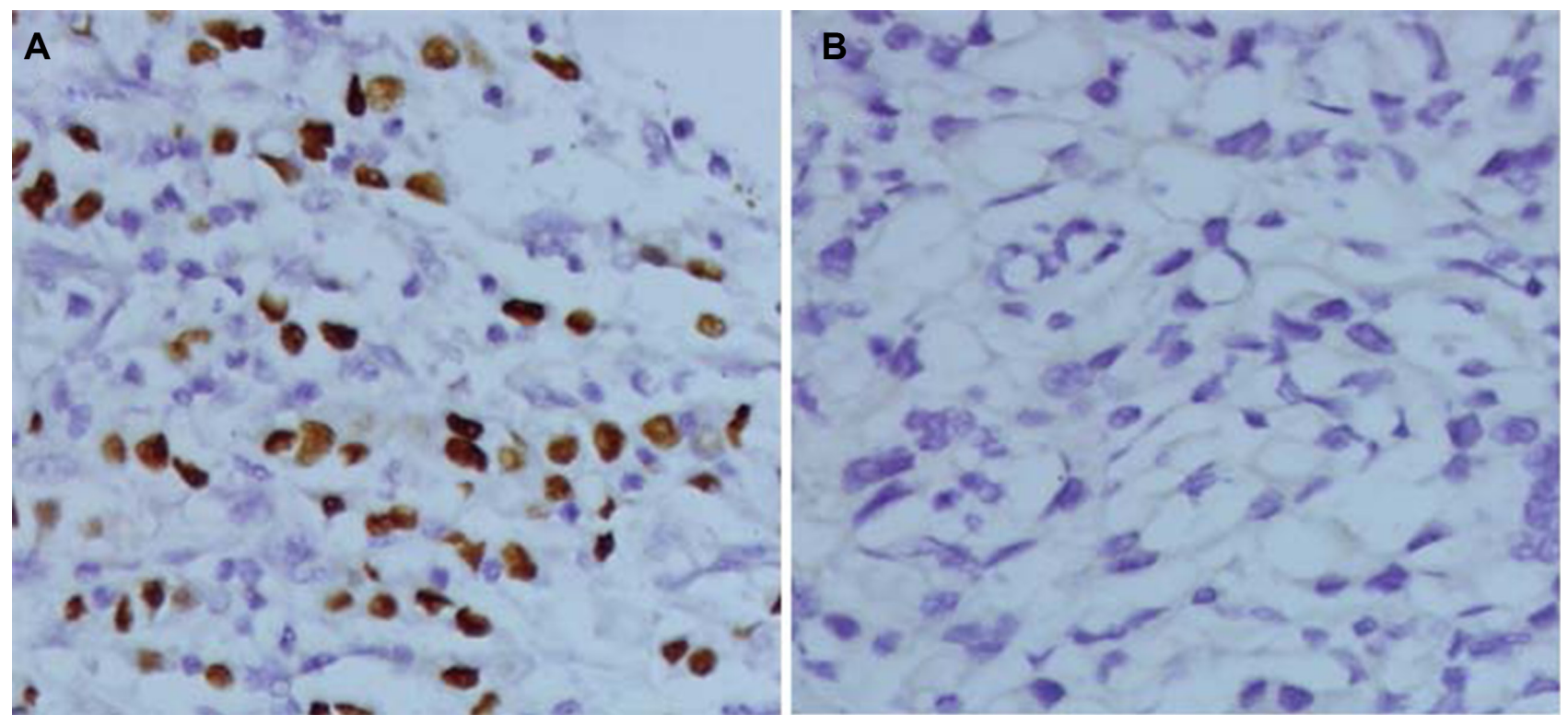

Figure 1: In situ hybridization of EBER1 in gastric cancer tissue. (A) EBVaGC, EBER(+) tissue. (B) EBVnGC, EBER(-) tissue. Magnification, ×400. EBER, EBV-encoded small RNA; EBVaGC, EBV-associated gastric carcinoma; EBVnGC, non-EBV-infected gastric cancer. 
EBVnGC and adjacent tissues. Next, we explored the target genes for EBV and SNHG8, and identified those that showed the same expression pattern: EBVaGC FPKM expression level $\geq 5$ and greater expression than in EBVnGC and in EBVaGC adjacent tissue (Table 4).

TRIM28, also known as KAP1 and TIF1b, is a universal co-repressor that mediates transcriptional control by interacting with Krüppel-associated box zinc finger proteins $[32,33]$. TRIM28 is an essential partner in several multiple-protein complexes and has a variety of functions including the regulation of pluripotency and proliferation $[34,35]$. It participates in epithelialmesenchymal transition via the regulation of histone acetylation and methylation on E- and N-cadherin promoters in lung cancer cell lines. TRIM28 is involved in cancer progression; it is overexpressed in colorectal and gastric cancer and is an independent prognostic factor for poor overall and relapse-free survival [36].
The highly conserved gene EIF4A2 is a member of the eukaryotic initiation factor 4A family, and encodes a protein synthesis initiation factor for binding mRNA to the ribosome. EIF4A2 is involved in the progression of breast cancer and melanoma [37] and in the development of non-small-cell lung cancer, and has been suggested as a potential prognostic marker [38].

Nucleosome assembly protein-1 (Nap1) plays a role in cell proliferation and cell cycle progression, as well as nucleosome assembly [39, 40]. Nap1-like 1 (Nap1L1) is highly homologous to Nap1 and shares some functions with it, such as nucleosome assembly, although it also has a more active role in nucleosome disassembly [40]. Nap1L1 is overexpressed in certain tumors such as hepatoblastoma [41] and carcinoid of the small intestine [42]. It epigenetically promotes tumor cell proliferation in pancreatic neuroendocrine neoplasms by inhibiting the tumor suppressor p57Kip2 and the mTOR pathway [43].

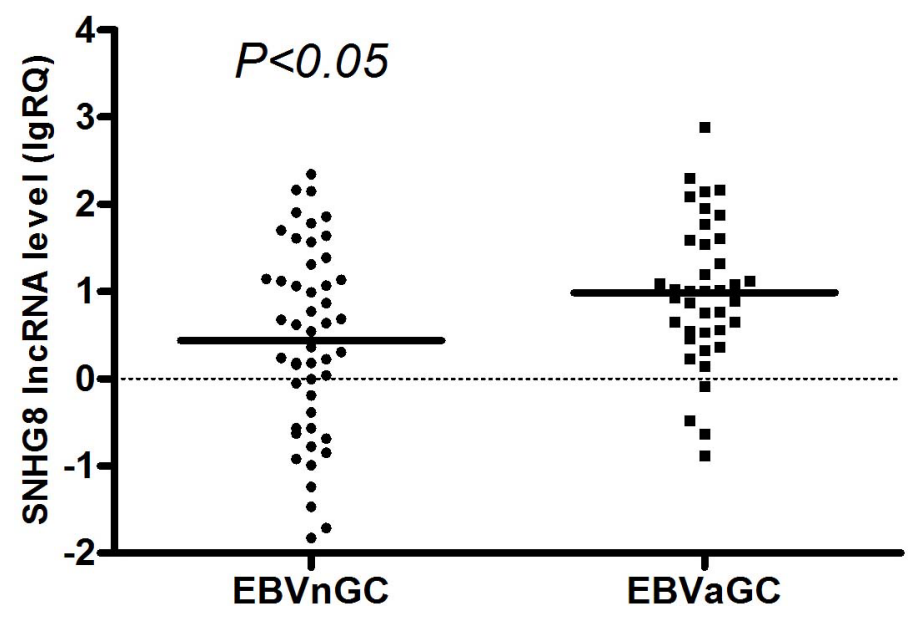

Figure 2: Distribution of SNHG8 IncRNA levels in EBVnGC and EBVaGC. Bold lines represent the mean value for each patient cohort; $R Q=2^{-\Delta \Delta C t}$

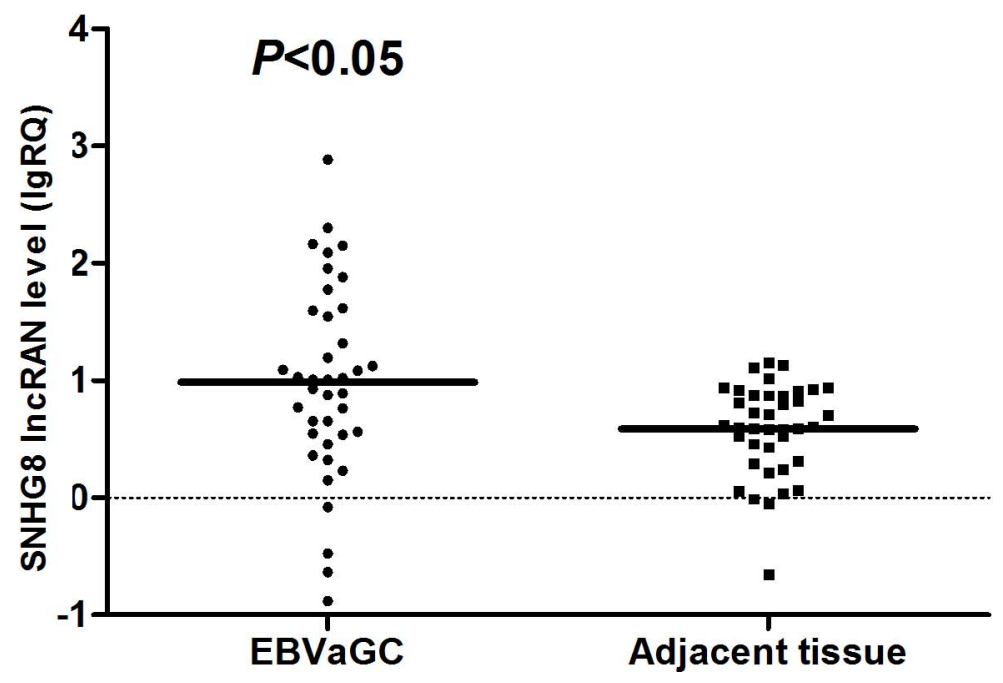

Figure 3: Distribution of SNHG8 IncRNA levels in EBVaGC and adjacent tissue. Bold lines represent the mean value for each patient cohort; $R Q=2^{-\Delta \Delta C t}$ 
Table 2: Significantly enriched KEGG and GO terms of SNHG8 target genes using DAVID

\begin{tabular}{|c|c|c|}
\hline Category & Term & FDR $(<0.05)$ \\
\hline KEGG PATHWAY & hsa03010:Ribosome & $3.77 \mathrm{E}-13$ \\
\hline \multirow{7}{*}{$\begin{array}{l}\text { Gene Ontology (GO) Biological } \\
\text { Processes (BP) }\end{array}$} & GO:0006412 translation & $1.54 \mathrm{E}-14$ \\
\hline & GO:0006414 translational elongation & $1.25 \mathrm{E}-11$ \\
\hline & GO:0006396 RNA processing & $2.91 \mathrm{E}-05$ \\
\hline & GO:0045449 regulation of transcription & 0.001145 \\
\hline & GO:0006350 transcription & 0.002024 \\
\hline & GO:0008380 RNA splicing & 0.040761 \\
\hline & GO:0016071 mRNA metabolic process & 0.041671 \\
\hline \multirow{5}{*}{$\begin{array}{l}\text { Gene Ontology (GO) Molecular } \\
\text { Function (MF) }\end{array}$} & GO:0003735 structural constituent of ribosome & $6.18 \mathrm{E}-09$ \\
\hline & GO:0003723 RNA binding & 2.84E-06 \\
\hline & GO:0008134 transcription factor binding & 0.002199 \\
\hline & GO:0003677 DNA binding & 0.004828 \\
\hline & GO:0030528 transcription regulator activity & 0.012988 \\
\hline \multirow{15}{*}{$\begin{array}{l}\text { Gene Ontology (GO) Cellular } \\
\text { Component (CC) }\end{array}$} & GO:0030529 ribonucleoprotein complex & $1.55 \mathrm{E}-13$ \\
\hline & GO:0022626 cytosolic ribosome & 5.93E-09 \\
\hline & GO:0005840 ribosome & $4.48 \mathrm{E}-08$ \\
\hline & GO:0043232 intracellular non-membrane-bounded organelle & 4.91E-07 \\
\hline & GO:0043228 non-membrane-bounded organelle & $4.91 \mathrm{E}-07$ \\
\hline & GO:0005829 cytosol & $2.56 \mathrm{E}-05$ \\
\hline & GO:0033279 ribosomal subunit & $9.32 \mathrm{E}-05$ \\
\hline & GO:0031981 nuclear lumen & $3.65 \mathrm{E}-04$ \\
\hline & GO:0022625 cytosolic large ribosomal subunit & $8.32 \mathrm{E}-04$ \\
\hline & GO:0044445 cytosolic part & 0.001145 \\
\hline & GO:0005730 nucleolus & 0.003792 \\
\hline & GO:0031974 membrane-enclosed lumen & 0.010549 \\
\hline & GO:0070013 intracellular organelle lumen & 0.013856 \\
\hline & GO:0043233 organelle lumen & 0.017307 \\
\hline & GO:0005681 spliceosome & 0.019806 \\
\hline
\end{tabular}

PLD3 encodes a lipase family protein associated with the endoplasmic reticulum, which is widely expressed in the brain, including in the hippocampus and most of the cortex $[44,45]$. PDL3 has been implicated in late-onset Alzheimer's disease and might contribute to a range of cellular functions including differentiation, epigenetic modification, neurotransmission, and signal transduction [44, 45].

Ribosomal protein L18a (RPL18A) is a component of the eukaryotic large ribosomal subunit (60S). RPL18A interacts with the hepatitis $\mathrm{C}$ virus internal ribosome entry site (IRES) and might be involved in IRES-mediated translation and viral replication $[46,47]$.

The channel kinase TRPM7 transduces physical and chemical stress. It has intrinsic kinase activity and is involved in cell growth, proliferation, migration, differentiation, and survival [48, 49]. Aberrant TRPM7 expression is associated with a number of cancers [49-51] including breast carcinoma and head/neck cancer [52-54]. Furthermore, TRPM7 might regulate exocrine pancreatic development, and aberrant TRPM7-mediated signaling contributes to the development of pancreatic cancer [54].

\section{DISCUSSION}

In the present study, we have evaluated the profiles of lncRNAs that are aberrantly expressed in EBVaGC, and confirmed expression levels of the lncRNA SNHG8 by RT-PCR. The putative functions of SNHG8 were explored by examining co-expression of lncRNA and mRNAs.

Mounting evidence suggests that lncRNAs, initially considered transcriptional noise, play pivotal roles in carcinogenesis $[11,55]$. In gastric cancer, the dysregulation of several lncRNAs is associated with tumorigenesis, 
Table 3: EBV proteins whose target genes significantly overlapped with SNHG8 targets

\begin{tabular}{|c|c|c|c|c|}
\hline $\begin{array}{c}\text { EBV } \\
\text { protein }\end{array}$ & $\begin{array}{c}\text { FDR } \\
(<0.05)\end{array}$ & $\begin{array}{l}\text { Number of } \\
\text { EBV target } \\
\text { genes }\end{array}$ & $\begin{array}{c}\text { Number of EBV } \\
\text { target genes that } \\
\text { were also targeted by } \\
\text { SNHG8 } \\
\end{array}$ & $\begin{array}{c}\text { EBV target genes that were also targeted by } \\
\text { SNHG8 }\end{array}$ \\
\hline LF3 & $6.93 \mathrm{E}-05$ & 300 & 28 & $\begin{array}{l}\text { AHDC1, AMBRA1, BAHD1, C19orf26, CENPB, } \\
\text { CIC, EEF2, EIF4A2, ELK1, GLTPD1, HNRNPA0, } \\
\text { IRF2BP1, KHSRP, KLHL26, MEF2D, MGRN1, } \\
\text { MLLT1, NCOR2, NFIC, PLD3, PLIN3, PTPN23, } \\
\text { SAMD4B, SART1, SF1, SURF6, ZBTB4, ZBTB7A }\end{array}$ \\
\hline BHLF1 & 0.000252 & 568 & 40 & $\begin{array}{l}\text { AHDC1, AMBRA1, BAD, BAHD1, BTBD2, BTF3, } \\
\text { C19orf26, CD58, CENPB, CIC, CLIP2, EEF2, EIF4A2, } \\
\text { ELK1, GLTPD1, GTF2F1, GTPBP1, HDGFRP2, } \\
\text { HNRNPA0, IRF2BP1, KHSRP, KLHL26, LARP7, } \\
\text { MEF2D, MLLT1, MLLT6, MTERFD3, N6AMT1, } \\
\text { NFIC, NUDT16L1, PLD3, PLIN3, SAMD1, SAMD4B, } \\
\text { SART1, SURF6, TAF7, TRIM28, ZBTB4, ZNF324B }\end{array}$ \\
\hline BHRF1 & 0.008401 & 793 & 45 & $\begin{array}{l}\text { AHDC1, BTBD2, BTF3L4, CD58, CENPB, CIC, } \\
\text { COMMD10, CPSF1, EEF2, EIF3G, EIF4A2, ERCC8, } \\
\text { GCNT2, GEN1, GLTPD1, GTF2F1, GTF2H2, GTPBP1, } \\
\text { HDGFRP2, IRF2BP1, KHSRP, KLHL26, MEF2D, } \\
\text { MGRN1, MLLT1, MTERFD3, NAP1L1, NCOR2, } \\
\text { NFIC, PLD3, PTPN23, RBM10, RNF14, SAMD1, } \\
\text { SAMD4B, SART1, TAF7, TMEM168, TRIM28, } \\
\text { TRPM7, ZBTB4, ZBTB7A, ZNF337, ZNF345, ZNF720 }\end{array}$ \\
\hline BNLF2a & 0.039096 & 40 & 6 & BRD4, DLGAP4, NFKBIL1, RPL18A, TRIP10, WBP2 \\
\hline
\end{tabular}

Table 4: The FPKM expression levels of EBV target genes with the same expression pattern as SNHG8

\begin{tabular}{|c|c|c|c|c|c|}
\hline Transcript ID & Transcript Name & $\begin{array}{c}\text { EBV-negative } \\
\text { tumor sample } \\
\text { (EBVnGC) }\end{array}$ & $\begin{array}{c}\text { EBV-negative } \\
\text { adjacent sample }\end{array}$ & $\begin{array}{c}\text { EBV-positive } \\
\text { tumor sample } \\
\text { (EBVaGC) }\end{array}$ & $\begin{array}{c}\text { EBV-positive } \\
\text { adjacent sample }\end{array}$ \\
\hline ENST00000600840 & TRIM28 & 3.38 & 5.18 & 5.18 & 3.55 \\
\hline ENST00000323963 & EIF4A2 & 0.67 & 16.67 & 21.20 & 18.62 \\
\hline ENST00000496382 & EIF4A2 & 0.00 & 0.00 & 5.61 & 0.00 \\
\hline ENST00000393263 & NAP1L1 & 1.48 & 2.70 & 5.53 & 3.65 \\
\hline ENST00000547773 & NAP1L1 & 1.81 & 1.47 & 5.29 & 2.06 \\
\hline ENST00000409281 & PLD3 & 5.57 & 0.00 & 11.27 & 0.00 \\
\hline ENST00000222247 & RPL18A & 74.79 & 150.44 & 101.37 & 81.57 \\
\hline ENST00000313478 & TRPM7 & 3.11 & 2.14 & 4.20 & 3.42 \\
\hline
\end{tabular}

metastasis, and prognosis $[12,56]$. For example, the Hox transcript antisense intergenic RNA (HOT-AIR), one of the most widely known lncRNAs, was shown to be associated with TNM stage and lymph node metastasis in patients with gastric cancer. HOT-AIR also promotes invasion and epithelial-mesenchymal transition by directing polycomb repressive complex 2 (PRC2) to silence HOXD9 [57, 58]. Homeobox A transcript at the distal tip (HOTTIP) is markedly overexpressed in gastric cancer tissues and associated with several measures of severity including TNM stage and overall survival. Furthermore, overexpression of HOTTIP was identified as an independent poor prognostic factor for patients with gastric cancer. Together, this indicates that lncRNAs are an excellent prospect as a new type of biomarker [56]. Our study also shows that SNHG8 expression was markedly elevated in EBVaGC tissues compared with normal control samples.

It has long been accepted that small nucleolar RNAs (snoRNAs) guide RNA in post-translational ribosomal RNA modification processes [59, 60]. However, 
accumulating evidence suggests that these non-coding RNAs might play a much more important role in cell fate determination and oncogenesis than previously thought $[61,62]$. In patients with gastric cancer, SNHG5 was significantly downregulated and associated with TNM stage [63]. Furthermore, SNHG20 was upregulated in hepatocellular carcinoma and, in an in vitro study, its suppression distinctly inhibited hepatocellular carcinoma cell proliferation, migration, and invasion [64]. SNHG8, located on $4 \mathrm{q} 26$, is thought to encode the smaller snoRNAs. Our study showed that SNHG8 expression in EBVaGC tissues is markedly elevated compared with normal control samples.

$\mathrm{EBV}$ is a lifelong latent infection present in more than $90 \%$ of the human population and has been linked etiologically to a wide range of human malignancies [65]. EBV-encoded proteins, such as EBV nuclear antigen 1 and latent membrane proteins, can alter gene expression, accelerate growth, increase survival, and facilitate invasion of transformed cells $[66,67]$. A number of viral non-coding RNAs have also been linked to latent EBV infection; for example, EBV BamHI-A rightward transcripts (BARTs), a family of alternatively spliced mRNAs expressed in EBV latency programs, are closely associated with clinical and pathological measures of tumor progression [68]. BART1 induces metastasis via PTEN-dependent pathway regulation [69]; BART3 promotes cell growth by its action on deleted in cancer 1 (DICE1) [70]; and BART5 inhibits apoptosis by modulating the pro-apoptotic protein p53 upregulated modulator of apoptosis (PUMA) [71]. This suggests that EBV plays a causal role in the development of malignancies, metastasis of tumors, and evasion of the host immune system.

EBVaGC has unique clinicopathologic characteristics, including better prognosis than EBVnGC. Several well-recognized viral genes such as $B H R F 1$, $B L L F 1, B R L F 1, B Z L F 1, E B N A 1$, and $L M P 2 A$ are highly expressed in EBVaGC $[72,73]$. Expression of LMP2A is involved in the upregulation of survivin protein and causes genome-wide aberrant methylation in host cells [74]. Patients with EBVaGC show typical genetic and epigenetic alterations, and approximately 205 host cell genes are usually mutated including AKT2, CCNA1, $M A P 3 K 4$, and TGFBR1 [74].

In our study, the lncRNA SNHG8 was expressed in an EBV-specific manner. SNHG8 expression in EBVaGC was higher than in EBVnGC and in EBVaGC adjacent tissue. Based on our analysis of SNHG8 and EBV targets, we propose a theory of how SNHG8 triggers gastric cancer (Figure 4). SNHG8 interacts with the EBV proteins LF3, BHLF1, BHRF1, and BNLF2a and regulates the expression of TRIM28, EIF4A2, NAP1L1, PLD3, RPL18A, and TRPM7. Functional analysis of TRIM28, EIF4A2, NAP1L1, PLD3, RPL18A, and TRPM7 suggested that they play direct roles in gastric cancer. This reveals the regulatory roles of 1 ncRNAs and viruses in gastric carcinoma, and contributes to a more comprehensive understanding of tumorigenesis.

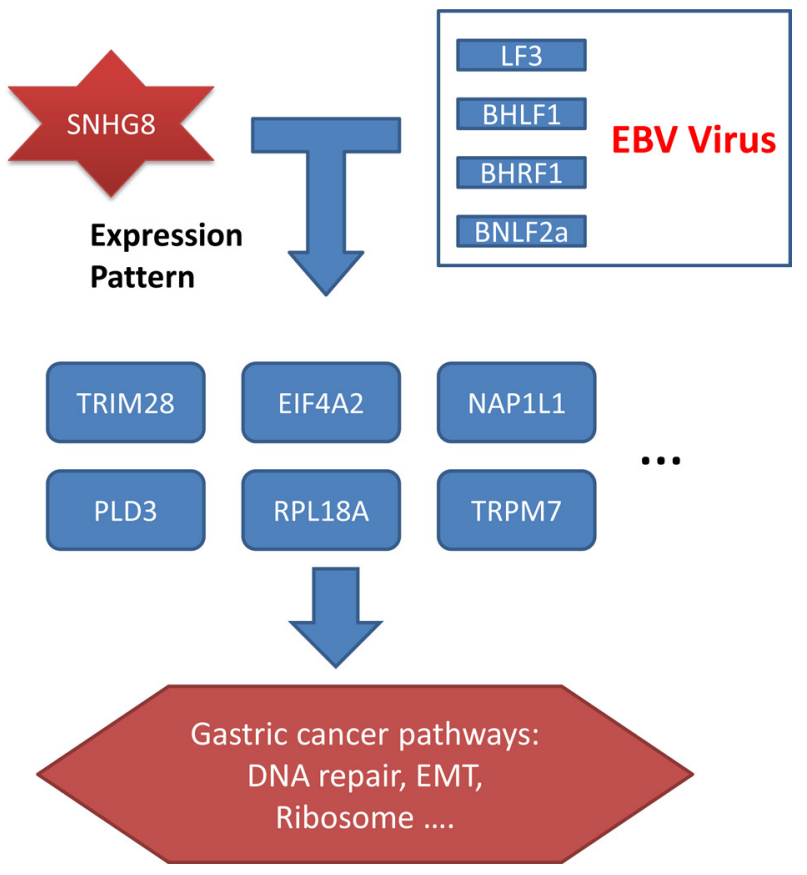

Figure 4: SNHG8, EBV, and their targets in EBV-associated gastric cancer. Co-expression and enrichment analysis showed that SNHG8 interacted with the EBV proteins LF3, BHLF1, BHRF1, and BNLF2a. These proteins in turn regulated the expression of TRIM28, EIF4A2, NAP1L1, PLD3, RPL18A, and TRPM7, which play important roles in gastric cancer pathways, contributing to processes such as DNA repair, epithelial-mesenchymal transition, and ribosomal function. 


\section{MATERIALS AND METHODS}

\section{Patient and tissue samples}

\section{RNA deep sequencing samples}

Two gastric cancer tissues (one EBVnGC and one EBVaGC) and their pair-matched adjacent gastric tissues were obtained from two patients at Fujian Provincial Cancer Hospital. The patients were male with poorly differentiated stage IIIB adenocarcinoma and lymph node metastasis.

\section{Validated samples}

Eighty-eight patients, including 39 with EBVaGC, were included in this study. They took gastric carcinoma resection from July 2012 to April 2015. The patients (69 males and 19 females) had a median age of 58.2 years (ranging from 22.0 to 79.0 years) and had a median tumor size of $5.5 \mathrm{~cm}(1.0-15.0 \mathrm{~cm})$. None of them received chemotherapy before surgery. Fresh stomach tumor tissues and their adjacent non-tumorous tissues were obtained immediately after tumor resection. One portion of the tissues was immediately snap-frozen in liquid nitrogen and then stored at $-80^{\circ} \mathrm{C}$; and the other portion was fixed in $10 \%$ buffered formalin and then embedded in paraffin. Lauren's criteria [75] was used to determine the histologic subtypes of the tumors. EBVaGC was identified by in situ hybridization for EBER1 (Dako, Denmark) (Figure 1) [76].

The study was approved by the Research Ethics Committee of the Fujian Provincial Cancer Hospital, China. Informed consent from all patients was obtained before participation.

\section{Sequencing and assembly}

Total RNA was isolated using Trizol reagent (Invitrogen, Carlsbad, CA, USA). RNA was examined by gel electrophoresis and only high quality RNA was used for subsequent analysis. RNA-Seq libraries were prepared using an Illumina HiSeq 3000 sequencing system with a 50 bp single-end protocol (Illumina, Inc., San Diego, CA, USA) [77]. In total, there were 52 and 59 million $2 \times 150$ paired-end reads of the paired GC/control mucosa RNA samples $[77,78]$.

The raw sequencing reads were analyzed with Trim Galore! Cutadapt wrapper v1.9.dev6 (http://www. bioinformatics.babraham.ac.uk/projects/trim_galore/) and Trimmomatic v0.32 [18]: the adapters were trimmed with the Trim Galore! Cutadapt wrapper using the -paired option, and low quality bases $(<\mathrm{Q} 20)$ were removed with Trimmomatic. FastQC v0.11.3 (http://www. bioinformatics.babraham.ac.uk/projects/fastqc/) was used to manually evaluate the qualities of the raw and trimmed sequencing data by checking per base quality, per base GC content and sequence length distribution. TopHat2 v2.1.1 [19] was used to align the trimmed reads to the human genome (GRCh37) with reference annotation from Gencode v19. More than $90 \%$ reads were mapped while over $85 \%$ reads were uniquely mapped. rRNA genes were masked, and transcriptome assemblies were generated using Cufflinks (version 2.2.1) [20].

\section{Criteria for defining EBV-specific IncRNAs}

EBV-specific lncRNAs were defined by fold changes ( $\geq 5$ ) between FPKM values of the EBV-positive tumor sample and the other three samples.

\section{Real-time RT-PCR assay}

Quantitative RT-PCR was used to validate the sequencing results. Total RNA from 88 paired gastric cancer and adjacent tissues was treated with DNaseI (Sigma, St Louis, MO, USA) to eliminate any genomic DNA contamination. Reverse transcription for lncRNAs was performed using M-MLV Reverse Transcriptase (Takara, Japan). The cDNA template was amplified by real-time RT-PCR using the SYBR Green Master Mix (Roche, USA). Primers were designed using Primer Premier 5.0 software. Real-time RT-PCR reactions were performed in triplicate on the ABI7500 system (Applied Biosystems, CA, USA).

Using the comparative $\mathrm{Ct}$ method $2^{-\Delta \Delta \mathrm{Ct}}[79,80]$ and gastric carcinoma sample No. 9 as a calibrator, the relative expression levels in all gastric carcinoma samples and adjacent non-tumorous tissues were quantified. Expression levels of lncRNA were normalized to $\beta$-actin mRNA expression.

\section{Identification of mRNA targets of IncRNAs}

To identify the mRNA targets of IncRNAs, we analyzed the RNA sequencing dataset of the Illumina Body Map [81], which included 16 normal human tissues. Expression levels of 14,886 lncRNAs from the LNCipedia database [82] and 21,721 protein-coding genes from UCSC hg19 [83] were calculated using TopHat [84] and Cufflinks [85] with default parameters. If the expression level of a protein-coding gene was correlated with that of a lncRNA with an absolute Pearson correlation coefficient $>0.5$, they were deemed a co-expression pair. The coexpressed mRNAs were considered to constitute the microenvironment around the lncRNA and were used to annotate the functions of the lncRNA.

\section{Identification of human target genes of EBV proteins}

The target genes of EBV proteins were obtained from EBV Genomics (https://ebv.wistar.upenn.edu) [86]. We downloaded the human gene expression levels and EBV expression levels in 201 samples, and then calculated 
the Pearson correlation coefficient between the human and EBV genes. Human genes with an absolute Pearson correlation coefficient $>0.5$ were considered as the target genes of an EBV gene.

\section{Enrichment between IncRNA and EBV}

Enrichment between lncRNA and EBV genes can be represented by the hypergeometric test $P$ value [87-89] of the target gene of lncRNA, L(i), and the target gene of EBV, $V(j)$, which can be computed by:

$$
\mathrm{p}(\mathrm{i}, \mathrm{j})=\mathrm{p}(\mathrm{L}(\mathrm{i}), \mathrm{V}(\mathrm{j}))=\sum_{k=m}^{n} \frac{\left(\begin{array}{l}
M \\
m
\end{array}\right)\left(\begin{array}{c}
N-M \\
n-m
\end{array}\right)}{\left(\begin{array}{l}
N \\
n
\end{array}\right)}
$$

where $N$ represents the total number of human genes, $M$ and $n$ represent the number of target genes of EBV gene $\mathrm{j}$ and the number of target genes of IncRNA i, respectively, and $m$ represents the number of lncRNA target genes that also target genes of EBV gene $\mathrm{j}$. The smaller the $P$ value for a lncRNA and an EBV gene, the stronger the suggested association between them. The hypergeometric test $P$ value was adjusted to the false discovery rate using the Benjamini method [90]. A false discovery rate of $<0.05$ was considered statistically significant.

\section{Statistical analysis}

Statistical analyses were conducted using IBM SPSS Statistics 19. The two-tailed Student's $t$ test was used to identify differentially expressed lncRNAs between EBVaGC and non-EBVaGC. $P<0.05$ was considered statistically significant.

\section{CONCLUSIONS}

Gastric cancer is an important malignancy with high morbidity and mortality rates and many risk factors. EBV is known to occur often in gastric cancer samples, but certain lncRNAs are also emerging as risk factors for cancer, although their precise roles in the disease remain unclear. To identify the key lncRNAs and investigate their functions and interactions with $\mathrm{EBV}$, we sequenced one EBVnGC tissue and its adjacent normal tissue, and one EBVaGC and its adjacent EBV-associated tissue. The lncRNA SNHG8 was expressed in an EBV-specific manner. Co-expression network analysis revealed significant interactions of SNHG8 and EBV LF3, BHLF1, $\mathrm{BHRF} 1$, and BNLF2a. Together, these factors regulate several functional genes in gastric cancer, such as TRIM28, EIF4A2, NAP1L1, PLD3, RPL18A, and TRPM7. This regulatory pathway model of IncRNA, virus, and target genes provides novel insights into gastric tumorigenesis and suggests potential drug targets for intervention.

\section{ACKNOWLEDGMENTS AND FUNDING}

This work was supported by the National Clinical Key Specialty Construction Program of China, the Medical Innovation Program of Fujian Province (2015CX-7) the Natural Science Foundation of Fujian Province (2016J0105), the Talent and Training Program of Fujian Provincial Health and Family Planning Commission (2013-ZQN-JC-8, 2015-ZQN-JC-7), the Shanghai Sailing Program, the Youth Innovation Promotion Association of the Chinese Academy of Sciences (2016245), and the Natural Science Foundation of Shanghai (16ZR1449900).

\section{CONFLICTS OF INTEREST}

The authors have declared no conflicts of interest.

\section{REFERENCES}

1. Torre LA, Bray F, Siegel RL, Ferlay J, Lortet-Tieulent J, Jemal A. Global cancer statistics, 2012. Cancer J Clin. 2015; 65:87-108.

2. Yau TO, Tang CM, Yu J. Epigenetic dysregulation in Epstein-Barr virus-associated gastric carcinoma: disease and treatments. World J Gastroenterol. 2014; 20:6448-6456.

3. Marquitz AR, Mathur A, Shair KH, Raab-Traub N. Infection of Epstein-Barr virus in a gastric carcinoma cell line induces anchorage independence and global changes in gene expression. Proc Natl Acad Sci USA. 2012; 109:9593-9598.

4. Cancer Genome Atlas Research N. Comprehensive molecular characterization of gastric adenocarcinoma. Nature. 2014; 513:202-209.

5. Kitagawa M, Kotake Y, Ohhata T. Long non-coding RNAs involved in cancer development and cell fate determination. Curr Drug Targets. 2012; 13:1616-1621.

6. Bartonicek N, Maag JL, Dinger ME. Long noncoding RNAs in cancer: mechanisms of action and technological advancements. Mol Cancer. 2016; 15:43.

7. Cao Y, Wang P, Ning S, Xiao W, Xiao B, Li X. Identification of prognostic biomarkers in glioblastoma using a long noncoding RNA-mediated, competitive endogenous RNA network. Oncotarget. 2016; 7:41737-41747. doi: 10.18632/ oncotarget.9569.

8. Zhan Y, Lin J, Liu Y, Chen M, Chen X, Zhuang C, Liu L, Xu W, Chen Z, He A, Zhang Q, Sun X, Zhao G, et al. Upregulation of long non-coding RNA PANDAR is associated with poor prognosis and promotes tumorigenesis in bladder cancer. J Exp Clin Cancer Res. 2016; 35:83.

9. Huang NS, Chi YY, Xue JY, Liu MY, Huang S, Mo M, Zhou SL, Wu J. Long non-coding RNA metastasis associated in lung adenocarcinoma transcript 1 (MALAT1) interacts with estrogen receptor and predicted poor survival in breast cancer. Oncotarget. 2016; 7:37957-37965. doi: 10.18632/oncotarget.9364. 
10. Nobili L, Lionetti M, Neri A. Long non-coding RNAs in normal and malignant hematopoiesis. Oncotarget. 2016; 7:50666-50681. doi: 10.18632/oncotarget.9308.

11. Schmitt AM, Chang HY. Long Noncoding RNAs in Cancer Pathways. Cancer cell. 2016; 29:452-463.

12. Fang XY, Pan HF, Leng RX, Ye DQ. Long noncoding RNAs: novel insights into gastric cancer. Cancer Lett. 2015; 356:357-366.

13. Nasrollahzadeh-Khakiani M, Emadi-Baygi M, Schulz WA, Nikpour P. Long noncoding RNAs in gastric cancer carcinogenesis and metastasis. Brief Funct Genomics. 2016.

14. Ma P, Xu T, Huang M, Shu Y. Increased expression of LncRNA PANDAR predicts a poor prognosis in gastric cancer. Biomed Pharmacother. 2016; 78:172-176.

15. Liu G, Xiang T, Wu QF, Wang WX. Long Noncoding RNA H19-Derived miR-675 Enhances Proliferation and Invasion via RUNX1 in Gastric Cancer Cells. Oncol Res. 2016; 23:99-107.

16. Malouf GG, Zhang J, Yuan Y, Comperat E, Roupret M, Cussenot O, Chen Y, Thompson EJ, Tannir NM, Weinstein JN, Valero V, Khayat D, Spano JP, et al. Characterization of long non-coding RNA transcriptome in clear-cell renal cell carcinoma by next-generation deep sequencing. Mol Oncol. 2015; 9:32-43.

17. Veneziano D, Nigita G, Ferro A. Computational Approaches for the Analysis of ncRNA through Deep Sequencing Techniques. Front Bioeng Biotechno. 2015; 3:77.

18. Bolger AM, Lohse M, Usadel B. Trimmomatic: a flexible trimmer for Illumina sequence data. Bioinformatics (Oxford, England). 2014; 30:2114-2120.

19. Kim D, Pertea G, Trapnell C, Pimentel H, Kelley R, Salzberg SL. TopHat2: accurate alignment of transcriptomes in the presence of insertions, deletions and gene fusions. Genome Biol. 2013; 14:R36.

20. Trapnell C, Roberts A, Goff L, Pertea G, Kim D, Kelley DR, Pimentel H, Salzberg SL, Rinn JL, Pachter L. Differential gene and transcript expression analysis of RNA-seq experiments with TopHat and Cufflinks. Nat Protocols. 2012; 7:562-578.

21. Huang da W, Sherman BT, Lempicki RA. Systematic and integrative analysis of large gene lists using DAVID bioinformatics resources. Nature protocols. 2009; 4:44-57.

22. Gruhne B, Kamranvar SA, Masucci MG, Sompallae R. EBV and genomic instability--a new look at the role of the virus in the pathogenesis of Burkitt's lymphoma. Semin Cancer Biol. 2009; 19:394-400.

23. Gruhne B, Sompallae R, Marescotti D, Kamranvar SA, Gastaldello S, Masucci MG. The Epstein-Barr virus nuclear antigen-1 promotes genomic instability via induction of reactive oxygen species. Proc Natl Acad Sci USA. 2009; 106:2313-2318.

24. Yoshioka M, Crum MM, Sample JT. Autorepression of Epstein-Barr virus nuclear antigen 1 expression by inhibition of pre-mRNA processing. J Virol. 2008; 82:1679-1687.

25. Xue SA, Jones MD, Lu QL, Middeldorp JM, Griffin BE. Genetic diversity: frameshift mechanisms alter coding of a gene (Epstein-Barr virus LF3 gene) that contains multiple 102-base-pair direct sequence repeats. Mol Cell Biol. 2003; 23:2192-2201.

26. Xue SA, Griffin BE. Complexities associated with expression of Epstein-Barr virus (EBV) lytic origins of DNA replication. Nucleic Acids Res. 2007; 35:3391-3406.

27. Huang Q, Petros AM, Virgin HW, Fesik SW, Olejniczak ET. Solution structure of the BHRF1 protein from EpsteinBarr virus, a homolog of human Bcl-2. J Mol Biol. 2003; 332:1123-1130.

28. Desbien AL, Kappler JW, Marrack P. The Epstein-Barr virus Bcl-2 homolog, BHRF1, blocks apoptosis by binding to a limited amount of Bim. Proc Natl Acad Sci USA. 2009; 106:5663-5668.

29. Hislop AD, Ressing ME, van Leeuwen D, Pudney VA, Horst D, Koppers-Lalic D, Croft NP, Neefjes JJ, Rickinson AB, Wiertz EJ. A CD8+ T cell immune evasion protein specific to Epstein-Barr virus and its close relatives in Old World primates. J Exp Med. 2007; 204:1863-1873.

30. Horst D, van Leeuwen D, Croft NP, Garstka MA, Hislop AD, Kremmer E, Rickinson AB, Wiertz EJ, Ressing ME. Specific targeting of the EBV lytic phase protein BNLF2a to the transporter associated with antigen processing results in impairment of HLA class I-restricted antigen presentation. J Immunol. 2009; 182:2313-2324.

31. Liu S, Wang X, Shu J, Zhao Z, Sun Z, Luo B. Sequence analysis of EBV immune evasion gene BNLF2a in EBV associated tumors and healthy individuals from nasopharyngeal carcinoma endemic and non-endemic regions of China. J Med Virol. 2015; 87:1946-1952.

32. Moosmann P, Georgiev O, Le Douarin B, Bourquin JP, Schaffner W. Transcriptional repression by RING finger protein TIF1 beta that interacts with the KRAB repressor domain of KOX1. Nucleic Acids Res. 1996; 24:4859-4867.

33. Kim SS, Chen YM, O’Leary E, Witzgall R, Vidal M, Bonventre JV. A novel member of the RING finger family, KRIP-1, associates with the KRAB-A transcriptional repressor domain of zinc finger proteins. Proc Natl Acad Sci USA. 1996; 93:15299-15304.

34. Iyengar S, Farnham PJ. KAP1 protein: an enigmatic master regulator of the genome. J Biol Chem. 2011; 286:26267-26276.

35. Chen L, Chen DT, Kurtyka C, Rawal B, Fulp WJ, Haura EB, Cress WD. Tripartite motif containing 28 (Trim28) can regulate cell proliferation by bridging HDAC1/E2F interactions. J Biol Chem. 2012; 287:40106-40118.

36. Fitzgerald S, Sheehan KM, O’Grady A, Kenny D, O'Kennedy R, Kay EW, Kijanka GS. Relationship between epithelial and stromal TRIM28 expression predicts survival in colorectal cancer patients. J Gastroenterol Hepatol. 2013; 28:967-974.

37. Yan LX, Wu QN, Zhang Y, Li YY, Liao DZ, Hou JH, Fu J, Zeng MS, Yun JP, Wu QL, Zeng YX, Shao JY. Knockdown of miR-21 in human breast cancer cell lines inhibits 
proliferation, in vitro migration and in vivo tumor growth. Breast Cancer Res : BCR. 2011; 13:R2.

38. Shaoyan X, Juanjuan Y, Yalan T, Ping H, Jianzhong L, Qinian W. Downregulation of EIF4A2 in non-small-cell lung cancer associates with poor prognosis. Clin Lung Cancer. 2013; 14:658-665.

39. Clark J, Alvarez DF, Alexeyev M, King JA, Huang L, Yoder MC, Stevens T. Regulatory role for nucleosome assembly protein-1 in the proliferative and vasculogenic phenotype of pulmonary endothelium. Am J Physiol Lung Cell Mol Physiol. 2008; 294:L431-439.

40. Okuwaki M, Kato K, Nagata K. Functional characterization of human nucleosome assembly protein 1-like proteins as histone chaperones. Genes Cells. 2010; 15:13-27.

41. Nagata T, Takahashi Y, Ishii Y, Asai S, Nishida Y, Murata A, Koshinaga T, Fukuzawa M, Hamazaki M, Asami K, Ito E, Ikeda $\mathrm{H}$, Takamatsu $\mathrm{H}$, et al. Transcriptional profiling in hepatoblastomas using high-density oligonucleotide DNA array. Cancer Genet Cytogenet. 2003; 145:152-160.

42. Kidd M, Modlin IM, Mane SM, Camp RL, Eick G, Latich I. The role of genetic markers--NAP1L1, MAGE-D2, and MTA1--in defining small-intestinal carcinoid neoplasia. Ann Surg Oncol. 2006; 13:253-262.

43. Schimmack S, Taylor A, Lawrence B, Alaimo D, SchmitzWinnenthal H, Buchler MW, Modlin IM, Kidd M. A mechanistic role for the chromatin modulator, NAP1L1, in pancreatic neuroendocrine neoplasm proliferation and metastases. Epigenetics \& chromatin. 2014; 7:15.

44. Zhang DF, Fan Y, Wang D, Bi R, Zhang C, Fang Y, Yao YG. PLD3 in Alzheimer's Disease: a Modest Effect as Revealed by Updated Association and Expression Analyses. Mol Neurobiol. 2015.

45. Cruchaga C, Karch CM, Jin SC, Benitez BA, Cai Y, Guerreiro R, Harari O, Norton J, Budde J, Bertelsen S, Jeng AT, Cooper B, Skorupa T, et al. Rare coding variants in the phospholipase D3 gene confer risk for Alzheimer's disease. Nature. 2014; 505:550-554.

46. Dhar D, Mapa K, Pudi R, Srinivasan P, Bodhinathan K, Das S. Human ribosomal protein L18a interacts with hepatitis $\mathrm{C}$ virus internal ribosome entry site. Arch Viro. 2006; 151:509-524.

47. Gramatikoff K, Schaffner W, Georgiev O. The leucine zipper of c-Jun binds to ribosomal protein L18a: a role in Jun protein regulation? Biol Chem Hoppe Seyler. 1995; 376:321-325.

48. Yee NS, Chan AS, Yee JD, Yee RK. TRPM7 and TRPM8 Ion Channels in Pancreatic Adenocarcinoma: Potential Roles as Cancer Biomarkers and Targets. Scientifica. 2012; 2012:415158.

49. Yee NS, Kazi AA, Yee RK. Cellular and Developmental Biology of TRPM7 Channel-Kinase: Implicated Roles in Cancer. Cells. 2014; 3:751-777.

50. Asrar S, Aarts M. TRPM7, the cytoskeleton and neuronal death. Channels. 2013; 7:6-16.
51. Norenberg W, Plotz T, Sobottka H, Chubanov V, Mittermeier L, Kalwa H, Aigner A, Schaefer M. TRPM7 is a molecular substrate of ATP-evoked P2X7-like currents in tumor cells. J Gen Physio. 2016; 147:467-483.

52. Guilbert A, Gautier M, Dhennin-Duthille I, Haren N, Sevestre H, Ouadid-Ahidouch H. Evidence that TRPM7 is required for breast cancer cell proliferation. Am J Physiol Cell Physiol. 2009; 297:C493-502.

53. Jiang J, Li MH, Inoue $\mathrm{K}$, Chu XP, Seeds J, Xiong ZG. Transient receptor potential melastatin 7-like current in human head and neck carcinoma cells: role in cell proliferation. Cancer Res. 2007; 67:10929-10938.

54. Yee NS, Zhou W, Liang IC. Transient receptor potential ion channel Trpm7 regulates exocrine pancreatic epithelial proliferation by $\mathrm{Mg} 2+$-sensitive Socs3a signaling in development and cancer. Dis Model Mech. 2011; 4:240-254.

55. Yang L, Lin C, Jin C, Yang JC, Tanasa B, Li W, Merkurjev D, Ohgi KA, Meng D, Zhang J, Evans CP, Rosenfeld MG. IncRNA-dependent mechanisms of androgen-receptor-regulated gene activation programs. Nature. 2013; 500:598-602.

56. Ye H, Liu K, Qian K. Overexpression of long noncoding RNA HOTTIP promotes tumor invasion and predicts poor prognosis in gastric cancer. Onco Targets Ther. 2016; 9:2081-2088.

57. Okugawa Y, Toiyama Y, Hur K, Toden S, Saigusa S, Tanaka K, Inoue Y, Mohri Y, Kusunoki M, Boland CR, Goel A. Metastasis-associated long non-coding RNA drives gastric cancer development and promotes peritoneal metastasis. Carcinogenesis. 2014; 35:2731-2739.

58. Liu YW, Sun M, Xia R, Zhang EB, Liu XH, Zhang ZH, Xu TP, De W, Liu BR, Wang ZX. LincHOTAIR epigenetically silences miR34a by binding to PRC2 to promote the epithelial-to-mesenchymal transition in human gastric cancer. Cell Death Dis. 2015; 6:e1802.

59. Matera AG, Terns RM, Terns MP. Non-coding RNAs: lessons from the small nuclear and small nucleolar RNAs. Nat Rev Mol Cell Biol. 2007; 8:209-220.

60. Jorjani H, Kehr S, Jedlinski DJ, Gumienny R, Hertel J, Stadler PF, Zavolan M, Gruber AR. An updated human snoRNAome. Nucleic Acids Res. 2016.

61. Liao J, Yu L, Mei Y, Guarnera M, Shen J, Li R, Liu Z, Jiang F. Small nucleolar RNA signatures as biomarkers for non-small-cell lung cancer. Mol Cancer. 2010; 9:198.

62. Martens-Uzunova ES, Jalava SE, Dits NF, van Leenders GJ, Moller S, Trapman J, Bangma CH, Litman T, Visakorpi T, Jenster G. Diagnostic and prognostic signatures from the small non-coding RNA transcriptome in prostate cancer. Oncogene. 2012; 31:978-991.

63. Zhao L, Guo H, Zhou B, Feng J, Li Y, Han T, Liu L, Li L, Zhang S, Liu Y, Shi J, Zheng D. Long non-coding RNA SNHG5 suppresses gastric cancer progression by trapping MTA2 in the cytosol. Oncogene. 2016. 
64. Assmann G, Schulte H. European lipid guidelines: therapeutic recommendations. European Atherosclerosis Society. Am J Cardiol. 1989; 63:53H-55H.

65. Kutok JL, Wang F. Spectrum of Epstein-Barr virusassociated diseases. Annu Rev Pathol. 2006; 1:375-404.

66. Wang L, Tian WD, Xu X, Nie B, Lu J, Liu X, Zhang B, Dong Q, Sunwoo JB, Li G, Li XP. Epstein-Barr virus nuclear antigen 1 (EBNA1) protein induction of epithelialmesenchymal transition in nasopharyngeal carcinoma cells. Cancer. 2014; 120:363-372.

67. Fang W, Zhang J, Hong S, Zhan J, Chen N, Qin T, Tang Y, Zhang Y, Kang S, Zhou T, Wu X, Liang W, Hu Z, et al. EBV-driven LMP1 and IFN-gamma up-regulate PD-L1 in nasopharyngeal carcinoma: Implications for oncotargeted therapy. Oncotarget. 2014; 5:12189-12202. doi: 10.18632/ oncotarget.2608.

68. Cai X, Schafer A, Lu S, Bilello JP, Desrosiers RC, Edwards R, Raab-Traub N, Cullen BR. Epstein-Barr virus microRNAs are evolutionarily conserved and differentially expressed. PLoS Pathog. 2006; 2:e23.

69. Cai L, Ye Y, Jiang Q, Chen Y, Lyu X, Li J, Wang S, Liu T, Cai H, Yao K, Li JL, Li X. Epstein-Barr virus-encoded microRNA BART1 induces tumour metastasis by regulating PTEN-dependent pathways in nasopharyngeal carcinoma. Nat Commun. 2015; 6:7353.

70. Lei T, Yuen KS, Xu R, Tsao SW, Chen H, Li M, Kok KH, Jin DY. Targeting of DICE1 tumor suppressor by Epstein-Barr virus-encoded miR-BART3* microRNA in nasopharyngeal carcinoma. Int J Cancer. 2013; 133:79-87.

71. Wan XX, Yi H, Qu JQ, He QY, Xiao ZQ. Integrated analysis of the differential cellular and EBV miRNA expression profiles in microdissected nasopharyngeal carcinoma and non-cancerous nasopharyngeal tissues. Oncol Rep. 2015; 34:2585-2601.

72. Sugiura M, Imai S, Tokunaga M, Koizumi S, Uchizawa M, Okamoto K, Osato T. Transcriptional analysis of EpsteinBarr virus gene expression in EBV-positive gastric carcinoma: unique viral latency in the tumour cells. Br J Cancer. 1996; 74:625-631.

73. Luo B, Wang Y, Wang XF, Liang H, Yan LP, Huang BH, Zhao P. Expression of Epstein-Barr virus genes in EBVassociated gastric carcinomas. World J Gastroenterol. 2005; 11:629-633.

74. Zhao J, Liang Q, Cheung KF, Kang W, Lung RW, Tong JH, To KF, Sung JJ, Yu J. Genome-wide identification of Epstein-Barr virus-driven promoter methylation profiles of human genes in gastric cancer cells. Cancer. 2013; 119:304-312.

75. Lauren P. The Two Histological Main Types Of Gastric Carcinoma: Diffuse And So-Called Intestinal-Type Carcinoma. An Attempt at a Histo-Clinical Classification. Acta pathologica et microbiologica Scandinavica. 1965; 64:31-49.
76. Iizasa H, Nanbo A, Nishikawa J, Jinushi M, Yoshiyama H. Epstein-Barr Virus (EBV)-associated gastric carcinoma. Viruses. 2012; 4:3420-3439.

77. Xiang JF, Yin QF, Chen T, Zhang Y, Zhang XO, Wu Z, Zhang S, Wang HB, Ge J, Lu X, Yang L, Chen LL. Human colorectal cancer-specific CCAT1-L lncRNA regulates longrange chromatin interactions at the MYC locus. Cell Res. 2014; 24:513-531.

78. Wang W, Wang X, Zhang Y, Li Z, Xie X, Wang J, Gao M, Zhang S, Hou Y. Transcriptome analysis of canine cardiac fat pads: involvement of two novel long non-coding RNAs in atrial fibrillation neural remodeling. J Cell Biochem. 2015; 116:809-821.

79. Lin X, Hu D, Chen G, Shi Y, Zhang H, Wang X, Guo X, Lu L, Black D, Zheng XW, Luo X. Associations of THBS2 and THBS4 polymorphisms to gastric cancer in a Southeast Chinese population. Cancer Genet. 2016; 209:215-222.

80. Lin XD, Chen SQ, Qi YL, Zhu JW, Tang Y, Lin JY. Overexpression of thrombospondin-1 in stromal myofibroblasts is associated with tumor growth and nodal metastasis in gastric carcinoma. J Surg Oncol. 2012; 106:94-100.

81. Farrell CM, O'Leary NA, Harte RA, Loveland JE, Wilming LG, Wallin C, Diekhans M, Barrell D, Searle SM, Aken B, Hiatt SM, Frankish A, Suner MM, et al. Current status and new features of the Consensus Coding Sequence database. Nucleic Acids Res. 2014; 42:D865-872.

82. Volders PJ, Helsens K, Wang X, Menten B, Martens L, Gevaert K, Vandesompele J, Mestdagh P. LNCipedia: a database for annotated human lncRNA transcript sequences and structures. Nucleic Acids Res. 2013; 41:D246-251.

83. Rosenbloom KR, Armstrong J, Barber GP, Casper J, Clawson H, Diekhans M, Dreszer TR, Fujita PA, Guruvadoo L, Haeussler M, Harte RA, Heitner S, Hickey G, et al. The UCSC Genome Browser database: 2015 update. Nucleic Acids Res. 2015; 43:D670-681.

84. Trapnell C, Pachter L, Salzberg SL. TopHat: discovering splice junctions with RNA-Seq. Bioinformatics. 2009; 25:1105-1111.

85. Trapnell C, Roberts A, Goff L, Pertea G, Kim D, Kelley DR, Pimentel H, Salzberg SL, Rinn JL, Pachter L. Differential gene and transcript expression analysis of RNA-seq experiments with TopHat and Cufflinks. Nat Protoc. 2012; 7:562-578.

86. Arvey A, Tempera I, Tsai K, Chen HS, Tikhmyanova N, Klichinsky M, Leslie C, Lieberman PM. An atlas of the Epstein-Barr virus transcriptome and epigenome reveals host-virus regulatory interactions. Cell host \& microbe. 2012; 12:233-245.

87. Huang T, Zhang J, Xu ZP, Hu LL, Chen L, Shao JL, Zhang L, Kong XY, Cai YD, Chou KC. Deciphering the effects of gene deletion on yeast longevity using network and machine learning approaches. Biochimie. 2012; 94:1017-1025. 
88. Chen L, Li B-Q, Feng K-Y. Predicting Biological Functions of Protein Complexes Using Graphic and Functional Features. Curr Bioinform. 2013; 8:545-551.

89. Yang J, Chen L, Kong X, Huang T, Cai Y-D. Analysis of Tumor Suppressor Genes Based on Gene Ontology and the KEGG Pathway. PLoS ONE. 2014; 9:e107202.
90. Benjamini Y, Hochberg Y. Controlling the False Discovery Rate-a Practical and Powerful Approach to Multiple Testing. J Roy Stat Soc B Met. 1995; 57:289-300. 